\title{
TRATMENT WITH FOLIC ACID INCREASES PARAOXONASE 1 ACTIVITY THEREBY IMPROVING CHRONIC KIDNEY DISEASE
}

(1) Andressa Keiko Matsumoto, (2,3,4) Michael Maes, (1) Ana Paula Michelin, (1) Kamila Landucci Bonifácio, (1) Laura de Oliveira Semeão, (1) Rafael Filipe Dal Ben Martins, (1) João Victor de Lima Pedrão, (1) Estefania Gastaldello Moreira, (5) Vinicius Daher Alvares Delfino, (1) Décio Sabbatini Barbosa.

(1) Health Sciences Graduate Program, Health Sciences Center, State University of Londrina, Londrina, PR, Brazil

(2) Department of Psychiatry, Faculty of Medicine, Chulalongkorn University, Bangkok, Thailand

(3) Department of Psychiatry, Medical University of Plovdiv, Plovdiv, Bulgaria

(4) IMPACT Strategic Research Center, Deakin University, Geelong, Australia

(5) Department of Internal Medicine, Nephrology Section, State University of Londrina, Londrina, PR, Brazil

Andressa Keiko Matsumoto: dessamatsu@hotmail.com

Michael Maes: dr.michaelmaes@hotmail.com

Ana Paula Michelin: paulimichelin10@gmail.com

Kamila Landucci Bonifácio: kamilalondrina@ hotmail.com

Laura de Oliveira Semeão: 1semeao @gmail.com

Rafael Filipe Dal Ben Martins: rfdalben@gmail.com

João Victor de Lima Pedrão: jvpedrao@gmail.com

Estefania Gastaldello Moreira: egmoreira22@hotmail.com 
Vinicius Daher Alvares Delfino: vddelfino@sercomtel.com.br

Decio Sabbatini Barbosa: sabbatini2011@hotmail.com 


\begin{abstract}
Introduction: Increased oxidative stress, including elevated homocysteine (Hcy) plasma levels, and lowered levels of antioxidants participate in the pathophysiology and progression of chronic kidney disease (CKD). Paraoxonase (PON)1 activity and folic acid are antioxidants which play a role in Hcy metabolism. However, there are no data whether, in CKD, treatment with folic acid improves glomerular filtration rate (GFR) through effects on PON1 activity and Hcy concentrations. Methods: In the current study, we determined PON1 genotypes and activity, Hcy and estimated GFR (eGFR) both before and after treatment with folic acid $(5 \mathrm{mg} / \mathrm{d})$ versus no treatment during three consecutive months in 113 outpatients with CKD classified into stages 4, 3b and 3a. Results: PON1 CMPAase and AREase activities were significantly lower in patients allocated to CKD stage 4 as compared with stages $3 \mathrm{~b}$ and $3 \mathrm{a}$. Treatment with folic acid significantly improved eGFR and increased levels of CMPAase and AREase in patients allocated to classes 4 and $3 \mathrm{~b}$, but not $3 \mathrm{a}$. The improvement of eGFR was associated with increased CMPAase and AREase activities, while the latter were associated with increased levels of folic acid. Treatment with folic acid significantly reduced plasma Hcy levels and the Hcy/PON1 activity ratio. The effects of folic acid increasing PON1 activities were not mediated by changes in Hcy. Discussion: Treatment of CKD patients in early/intermediate stages of CKD patients improves oxidative stress by rebalancing the prooxidant (Hcy) / antioxidant (PON1 activities) ratio. Treatment with folic acid significantly improves eGFR and these effects are mediated via increased PON1 activities. Treatment with folic acid in phase G3b and G4 may reduce renal disease progression by enhancing antioxidant defenses.
\end{abstract}


Keywords: chronic kidney disease, folic acid, inflammation, oxidative stress, antioxidants, biomarkers. 


\section{Introduction}

Chronic kidney disease (CKD) is a serious public health issue with an incidence that is still increasing (Neuen et al., 2017). In the Global Burden of Disease Study (GBD 2015), CKD was the 12th most common cause of death, accounting for 1.1 million deaths worldwide. CKD is defined as renal dysfunction with an estimated glomerular filtration rate [eGFR] $<60 \mathrm{~mL} / \mathrm{min} / 1.73 \mathrm{~m} 2$ and which should be present for at least 3 months (KDIGO, 2012). According to the National Kidney Foundation (KDIGO, 2012), staging of CKD is based on eGFR values whereby G1 stage refers to normal (eGFR $\geq 90 \mathrm{~mL} / \mathrm{min} / 1.73 \mathrm{~m} 2$ ), G2 to mildly decreased $(\mathrm{eGFR}=60-89 \mathrm{~mL} / \mathrm{min} / 1.73 \mathrm{~m} 2), \mathrm{G} 3 \mathrm{a}(\mathrm{eGFR} 59-44 \mathrm{~mL} / \mathrm{min} / 1.73$ $\mathrm{m} 2$ ), G3b (eGFR 44-33 mL/min/1.73 m2), G4 to severely decreased kidney function (eGFR 29-15 $\mathrm{mL} / \mathrm{min} / 1.73 \mathrm{~m} 2$ ), and G5 to kidney failure including end-stage renal disease (ESRD) (eGFR $<15 \mathrm{~mL} / \mathrm{min} / 1.73 \mathrm{~m} 2)$.

Age, hypertension, smoking, dyslipidemia and diabetes mellitus are some of the known risk factors of CKD (Aslam 2008; Levi et al., 2014). Many CKD-provoking factors are associated with mitochondrial dysfunctions and increased nitro-oxidative stress (Levi et al., 2014). Oxidative stress (OS) occurs in the early stages of CKD and increases with progression of renal impairment and further increases in end-stage renal disease (ESRD) (Oberg et al., 2004). Activated OS pathways are even more disturbed in patients undergoing renal replacement therapies due to procedure-related factors (Dounousi et al., 2006). Uremic toxins, including urea, creatinine, homocysteine [Hcy], oxalate, phosphorus, and guanidines, may promote lipid peroxidation and protein oxidation and generate an imbalance in the ratio of oxidized to reduced glutathione and reduced superoxide dismutase / peroxidase activity (Barreto et al., 2014; Stępniewska 2015; Duni et al., 2017; Taylor, Bhandari, Seymour 2015).

Hcy, formed from hepatic methionine, is metabolized by Hcy-thiolactonase (HTLase), which hydrolyzes Hcy-thiolactone to Hcy (Yilmaz, 2010). Hcy is minimally 
eliminated by the kidney since, in physiological conditions, only non-protein bound Hcy is subjected to glomerular filtration and reabsorbed in the tubuli and oxidized to carbon dioxide and sulfate by kidney cells (Perna et al., 2010). Moreover, in the kidney, Hcy is transsulfurated and a deficiency of this renal transsulfuration mechanism may contribute to elevated plasma Hcy (Long; Nie 2016). Increased Hcy levels are frequently observed in the plasma of CKD patients and are, additionally, associated with elevated OS (Kennedy et al., 2013). Moreover, plasma Hcy is modulated by genetic alterations in methionine metabolism enzymes, and deficiencies in vitamin B12 and B6 and folic acid (Long; Nie 2016). Folic acid and vitamin B12 are essential cofactors in the methylation of Hcy into methionine and, therefore, disruption of their homeostasis may be associated with increased cardiovascular risk and CKD progression (Cianciolo et al., 2008, 2017).

The metabolism of 5-methyltetrahydrofolate (5-MTHF), the circulating form of folic acid, is impaired in uremic patients (Pietrzik, Bailey, Shane 2010) including a slower uptake rate of folates into tissues (Zha, 2017). Folic acid supplementation at doses of 2.5 to $15 \mathrm{mg}$ may be efficient in reducing Hcy serum levels in CKD pre-dialysis or hemodialysis patients (Righetti et al., 2006; Vianna et al., 2007; Cianciolo et al., 2008; House et al., 2010; Wu, 2012). Moreover, there is evidence that folic acid exerts both direct and indirect antioxidant effects, such as free radical scavenging, protection against oxidative modification of human low-density lipoproteins, and improvement of cellular antioxidant defenses (Joshi et al., 2001; Nakano; Higgins; Powers 2001). Doshi et al. (2002) suggested that 5-MTHF may attenuate Hcy-induced production of superoxide radicals in cultured endothelial cells.

In CKD, elevated nitro-oxidative stress is mainly the consequence of increased production of reactive oxygen and nitrogen species (ROS / RNS) as a consequence of reduced clearance of pro-oxidant substances due to renal dysfunction while impaired antioxidant defenses may increase vulnerability to oxidative damage (Locatelli et al., 2003). 
One of those antioxidant enzymes is paraoxonases (PON)1 a high-density lipoprotein (HDL) associated enzyme displaying arylesterase (AREase) and lactonase activity (Costa, Giordano, Furlong 2011). PON1 protects lipids, HDL and LDL cholesterol from oxidation thereby protecting against the consequences of lipid peroxidation including cardiovascular disorder (Efrat; Aviram 2008; Rosenblat et al., 2011). Moreover, PON1 may exhibit a same activity as HTLase thereby hydrolyzing Hcy-thiolactone back to Hcy, which in turn may be converted to methionine (Yilmaz et al., 2006), thereby protecting against Hcy-associated ROS and OS (Yilmaz 2010). The PON1 Q192R polymorphism influences the activity of the PON1 enzymes thereby altering their ability to prevent lipid oxidation (Billecke et al., 2000). RR homozygotes show a greater efficacy detoxifying substrates including paraoxon, 4(chloromethyl)phenylacetate (CMPA) and 5-thiobutil butyrolactone (Richter et al., 2008; Marsillach et al., 2009; Bayrak et al., 2016). Importantly, Kenned et al. (2013) reported that PON activity may be lowered in CKD patients. Nevertheless, there are no data whether treatment with folic acid may improve PON1 activity in CKD patients and whether this could result in improvement of GFR through effects on Hcy.

Hence, the current study was carried out to examine whether, in pre-dialysis patients (stages G3a, G3b and G4), folic acid 5 mg/daily may enhance PON1 enzyme activity and improve eGFR and Hcy levels. The specific hypotheses are that a) folic acid may enhance PON1 activity in CKD patients; and b) that this effect could improve eGFR and lower Hcy levels.

Participants and Methods

Participants

In this study, 113 outpatients with CKD were recruited at the Department of Internal Medicine, Nephrology Section, the State University Clinical Hospital of Londrina (HC- 
UEL). All participants were Brazilian nationals, aged 18-65 years, both women and men. The clinical diagnosis of CKD was made by senior nephrologists based on eGFR values $<60$ $\mathrm{mL} / \mathrm{min} / 1.73 \mathrm{~m}^{2}$ and presence of markers of kidney damage including albuminuria, aberrations in urine sediment and electrolyte abnormalities. The Modification of Diet in Renal Disease (MDRD) Study equation (Levey et al., 1999) was used to estimate eGFR based on serum creatinine levels. CKD patients were classified into three groups according to eGFR classification G3a, G3b and G4.

Socio-demographic and clinical data were collected from all participants including presence of diabetes, hypertension, and use of statins and antihypertensive. Systolic and diastolic blood pressure (SP and DP) was measured using aneroid sphygmomanometer. The diagnosis of tobacco use disorder (TUD) was made using DSM-IV-TR criteria. We also measured body mass index (BMI) as body weight $(\mathrm{kg}) /$ length (m2).

Exclusion criteria for all subjects were a) age less than 18 years; b) neuroinflammatory disorders including Parkinson's disease, stroke, and multiple sclerosis; c) medical illness including malignancies; and d) use of immunomodulatory drugs, and antioxidant and $\omega 3$-polyunsaturated fatty acid supplements. We also excluded patients who did not comply with the G4, G3b and G3a staging criteria.

This is a nonblinded randomized trial, which examines the effects of intervention with folic acid on PON1 activities, Hcy and eGFR. Patients were allocated (simple randomization at a ratio 6/4) into an active intervention group (treatment with folic acid) or a control group (no active intervention). Treatment with folic acid was fixed at a dose of 5 $\mathrm{mg}$ in a tablet daily for 3 consecutive months. The choice of this dosage is based on previous studies (Nanayakkara et al., 2007; House et al., 2010). All patients were treated with care as usual (CAU), that is use of antidiabetics ( $n=29 / 23)$, statins $(n=9 / 10)$, antihypertensive drugs $(n=18 / 16)$ and acetylsalicylic acid $(n=35 / 29)$ in the intervention and control group, 
respectively. These treatments and their dosages were stable for at least 8 weeks prior to the initiation of the trial and during the study as well. No other treatments were started during the study period. All patients had blood tests at baseline and again 3 months later. After informed consent was given, the participants underwent clinical interviews and on the same day blood samples were collected while the intervention started the next day in the morning. A post-discontinuation interview and blood collection was conducted 12-weeks posttreatment. All patients from the extended intervention group had follow-up calls once a week. Treatment compliance was ascertained via pill counts and self-reporting.

Approval for the study was obtained from the Human Research Ethics Committee of the State University of Londrina (UEL), Brazil (CAAE 63097216.1.0000.5231). All participants provided written informed consent before participating in this study. This study was registered in the Brazilian Record of Clinical Trials (ReBEC), under n RBR-2bfthr.

Assays

Fasting blood was sampled from all individuals between 8.00 and 9.00 a.m. Blood was immediately centrifuged, and the serum was aliquoted and stored at $-80^{\circ} \mathrm{C}$ until thawed for assays. To stratify individuals in the functional genotypes for the PON1 Q192R polymorphism (QQ, QR, and RR), the substrates used were phenylacetate (PA, Sigma, USA) under high salt condition and 4-(chloromethyl)phenylacetate (CMPA, Sigma, USA), which is an alternative to the use of the toxic paraoxon. PON1 activities were determined by the rate of hydrolysis of CMPA (CMPAase, which is influenced by the PON1 Q192R polymorphism) as well as by the rate hydrolysis of phenylacetate under low salt condition (AREase, which is less influenced by the PON1 Q192R polymorphism). Analysis were conducted in a microplate reader (EnSpire, Perkin Elmer, USA) (Richter et al., 2008). Analyses of folate and serum creatinine were performed on the Dimension®, RxL apparatus 
(Deerfield, IL, USA). Hcy was measured by automated methodologies on the Architect i2000SR apparatus (Abbott, IL, USA). The intra-assay CV values for all analytes were $<10 \%$.

Statistical analysis

Analysis of contingency tables $\left(\chi^{2}\right.$ test) was employed to assess the associations between categorical variables while analysis of variance (ANOVA) was employed to assess differences in continuous variables between treatment groups. Generalized estimating equation (GEE) analysis, repeated measures, was employed to assess the effects of folic acid on the eGFR and biomarkers. The pre-specified GEE analyses included categorical effects of folic acid treatment (yes/no), time and the time-by-treatment interaction. GEE analysis, repeated measures, was also used to examine the changes over time (from baseline to endpoint) in eGFR and biomarker data. All tests were two-tailed and a p-value of 0.05 was used for statistical significance. The statistical analyses were performed using IBM SPSS windows version 25 . Two z unit-weighted composite scores were computed, a first reflecting an integrative index of PON1 activity computed as z score of CMPAase activity $+\mathrm{z}$ score of AREase activity, and the second reflecting a PROOX/ANTIOX ratio computed as z score of Hcy - z score of (zCMPAase + zAREase).

\section{Results}

Consort flow diagram

Figure 1 shows the CONSORT flow diagram and the progress of the paraticiants through the randomized trial. Of the 209 CKD patients included into the study, 73 were excluded due to different exclusion criteria as shown in Figure 1. As a consequence, one hundred thirty six participant were randomized in the study namely $82(60.30 \%)$ patients 
were allocated to the intervention arm treated with folic acid and CAU, and 54 (39.70\%) patients were allocated to the CAU only or control group. After randomization, 23 patients were excluded, i.e. 16 in the intervention and 7 in the control arm (see Figure 1). Finally, 113 patients completed the 12-weeks follow-up assessment and we analyzed the data of 66 partients treated with folic acid + CAU and 47 with CAU alone using a classical intentionto-treat-analysis. There were no missing data in both treatment arms either at baseline or after treatment.

\section{Baseline characteristics of patients in both treatment arms}

Table 1 shows the baseline characteristics of CKD patients treated with and without folic acid. There were no significant differences between both treatment groups in any of the demographic data including age, sex, and ethnicity and baseline clinical data including BMI, diabetes, use of statins, hypertension, use of antihypertensive, diastolic blood pressure (DP), and smoking. The systolic blood pressure was somewhat higher in patients treated with folic acid than in those without. There were no significant differences in any of the baseline biomarker levels between both treatment groups including in folic acid, eGFR, CMPAase, AREase, Hcy and vitamin B12 levels. Also, there were no significant differences in PON1 genotype distribution and eGFR grouping between patients who were treated or not with folic acid.

\section{Effects of treatment with folic acid}

Table 2 shows the results of GEE analysis, repeated measures, namely the effects of treatment with folic acid on the eGFR and biomarkers. We examined the effects of time (baseline versus 3 months later), treatment (study groups with or without folic acid), and the time $\mathrm{X}$ treatment group interaction. The first GEE analysis in Table 2 shows a significant 
effect of treatment, time, and the interaction pattern between time $\mathrm{X}$ treatment on the eGFR values, namely post-treatment eGFR values were significantly higher in patients treated with folic acid as compared with the three other conditions. The time $\mathrm{X}$ treatment interactions were also significant for folic acid, PON1 CMPAase and AREase activities, Hcy, and the PROOX/ANTIOX ratio. Post-treatment levels of folic acid, CMPAase and AREase were significantly increased in patients treated with folic acid as compared with their baseline levels and the pre- and post-treatment levels measured in patients who were not treated with folic acid. The post-treatment levels of Hcy and the PROOX/ANTIOX ratio were significantly lower in patients treated with folic acid as compared with their baseline levels and baseline and endpoint levels measured in patients who were not treated with folic acid. There were no significant effects of treatment, time or their interaction on vitamin B12 concentrations.

\section{Associations between changes in folic acid, GFR, PON1 activities and Hcy}

Employing GEE analysis, repeated measures, we examined the associations between the changes over time in eGFR, PON1 activities, and Hcy (introduced as dependent variables) and folic acid concentrations (introduced as explanatory variable). We found that the changes in folic acid from baseline to 3 months later were significantly and positively correlated with the changes in eGFR and PON1 CMPAase and AREase activities and inversely with the changes in Hcy. Since increased levels of Hcy may result in part from lowered eGFR we have also examined the combined effects of folic acid and eGFR on Hcy levels and found that both folic acid and eGFR significantly and inversely predicted Hcy levels (Table 3).

We also found that the eGFR values measured at baseline and 3 months later were significantly and positively correlated with the PON1 CMPAase and AREase activities. 
Moreover, the significant association between eGFR values and the time $\mathrm{X}$ folic acid treatment interaction (see Table 2) disappeared after considering the effects of PON1 enzyme activities, indicating that the time $\mathrm{X}$ folic acid treatment effect is mediated by increments in PON1 activities. Finally, Hcy did not have any significant effects on PON1 enzyme activities after considering the effects of folic acid.

\section{Effects of GFR groups}

Table 4 shows the differences in PON1 enzyme activities and Hcy and their ratio between the three groups divided according to eGFR values into groups G3a, G3b and G4. PON1 CMPAase and AREase activities were significantly lower in group G4 than in groups G3b and G3a. Hcy levels were significantly different between the three study samples with the lowest levels in group G3a and the highest levels in group G4. There were highly significant differences in the PROOX/ANTIOX ratio among the three groups with the highest levels in group G4 and the lowest levels in group G3a with a difference between those groups of around 1.475 standard deviations.

GEE analysis, repeated measures, with PON1 activities as dependent variable and time, folic acid treatment, and eGFR groups (G3a, G3b and G4) and their two- and threeway interactions as effects showed a significant three-way interaction between time by treatment by eGFR groups. Table 5 shows this interaction pattern, namely that in patients treated with folic acid, the post-treatment PON1 activities are significantly higher than the baseline values in groups G4 and G3b, but not in group G3a, while no significant effects were established in patients without active treatment indicating that treatment with folic acid increases PON1 activities only in patients belonging to groups G4 and G3b, but not group G4. 


\section{Discussion}

The first major finding of this study is that treatment with folic acid $5 \mathrm{mg}$ daily for three consecutive months significantly improved eGFR increasing the pre-treatment mean eGFR value of $36.8(\mathrm{SE}=0.5) \mathrm{ml} / \mathrm{min} / 1.73 \mathrm{~m}^{2}$ to a post-treatment value of $42.3(1.3)$ $\mathrm{ml} / \mathrm{min} / 1.73 \mathrm{~m}^{2}$. These findings extend those of a previous randomized, double-blind trial performed on $93 \mathrm{CKD}$ patients, who were not on dialysis, reporting that supplementation with folic acid $5 \mathrm{mg} / \mathrm{d}(6,12,18$ and 24 months) induced a moderate increase in eGFR levels although the overall effect was not significant (Nanayakkara et al., 2007). However, the results of other folate interventional studies are conflicting and some studies even reported a decrease in eGFR after treatment with different doses $(0.8 \mathrm{mg} / \mathrm{d}$ and $2.5 \mathrm{mg} / \mathrm{d})$ of folic acid in a single tablet containing high doses of combined B vitamins (folic acid, vitamin B6, and vitamin B12) in patients with advanced CKD (House et al., 2010). The latter authors explained these findings by postulating a potential toxicity determined by unmetabolized folic acid accumulating in the bloodstream. Nevertheless, the contradictory results may be consequence of differences in dose namely $5 \mathrm{mg}$ daily in the studies that reported an improvement in eGFR versus $<5 \mathrm{mg}$ daily in the negative studies. Even more important is a possible difference in CKD subgroups among studies. Thus, both above-mentioned studies reporting positive effects included patients with who were not treated with dialysis, whereas House et al. (2010) included dialysis patients. Another interpretation of the conflicting results is that the greater decrease in GFR in the House et al. (2010) study may be caused by toxicity induced by use of high vitamin B6 doses (Zempleni and Kiibler, 1995). Because these vitamins are water-soluble and renally excreted, vitamin B 6 toxicity may be more of a concern in patients with impaired renal function (House et al., 2010). Our results also extend the report of Xu et al. (2016) who found a small but statistically significant reduction in CKD progression with addition of folic acid to enalapril in adults with hypertension. 
Treatment with $10 \mathrm{mg}$ enalapril combined with $0.8 \mathrm{mg}$ folic acid significantly delayed the progression of CKD among patients with mild-to-moderate CKD as compared with $10 \mathrm{mg}$ enalapril alone. Wyatt and Spence (2016) pointed out that differences between Xu's study (2016) and prior studies may be explained by differences in study population namely folic acid fortification of grain, which is not implemented in China but is implemented in other countries (including the USA and Brazil) with the aim to reduce the risk of neural tube defects. Therefore, our results show that folic acid may improve eGFR also in patients with mandatory folic acid fortification.

The second major finding of this study is that PON1 CMPAase and AREase activities were significantly lowered in patients in stage G4 as compared with stages G3b and G3a. Our results extend those of Karatas et al. (2019) who reported that PON1 levels decreased with the increasing severity of CKD stages. Lowered levels of PON activity are frequently associated with CKD. For example, AREase activities were significantly lowered in CKD patients as compared with non-CKD patients while lowered activity levels also predicted a poorer outcome (Kennedy et al., 2013). Another study reported lowered levels of both paraoxonase and AREase in CKD and ESRD patients as compared with controls (Miljkovic et al., 2018).

Total PON1 activity was significantly reduced in hemodialysis patients as compared with controls and renal failure patients (Samouilidou et al., 2016). Furthermore, in CKD, PON1 AREase activity is significantly and negatively associated with creatinine concentrations (Miljkovic et al., 2018). Such findings indicate that PON1 enzyme activities are significantly reduced in parallel with increasing CKD severity with lowest levels being established in ESRD. Therefore, increasing severity of CKD appears to be accompanied by lowered total PON1 CMPAase and AREase activities and, consequently, lower lipid- 
associated antioxidant defenses and an increased vulnerability to develop damage due to OS (Moreira et al., 2019).

In this respect, Stefanovic et al. (2017) reported that the ratio of myeloperoxidase (MPO) / PON1 is significantly elevated in CKD patients as compared with controls while this ratio was inversely associated with smaller HDL classes. In addition, PON1 activities were negatively associated with levels of malondialdehyde (Atamer et al., 2008). In CKD patients, lowered PON1 concentrations are positively associated with the levels of HDL and HDL3 cholesterol and with ApoA1 (Samouilidou et al., 2016). Interestingly, lowered AREase activity predicted an increased risk towards adverse cardiac effects including myocardial infarction, stroke, and death (Kennedy et al., 2013).

Some studies show that OS is present even at the early stages of CKD and increases in parallel with progression of CKD to achieve very high levels in ESRD (Dounousi et al., 2006; Liakopoulos et al., 2019). There is now evidence that lowered antioxidant defenses and oxidative damage together with other inflammatory mediators play a key role in the onset of CKD, glomerular lesions, kidney disease progression, and CKD complications including inflammatory processes, atherosclerosis and hypertension (Daenen et al., 2019). The latter authors review that the kidney is vulnerable to oxidative damage because it is a highly metabolic organ that is rich in mitochondrial oxidative reactions. All in all, our results indicate that lowered PON1 CMPAase and AREase activities may be a key component in the pathophysiology of CKD by aggravating the damage due to OS.

The third major finding of this study is that treatment with folic acid significantly increased levels of CMPAase and AREase especially in patients allocated to CKD classes G4 and G3b, but not G3a. Only few studies examined the effects of folic acid on PON1 enzymatic activities and the few who reported negative findings. Firstly, Vanzin et al. (2015) reported no effects of folic acid on PON1 activity levels in patients with cystathionine- 
synthase deficiency. Secondly, in coronary artery disease patients, administration of folic acid for one year did not affect PON1 activities and PON1 protein (Perła-Kaján et al., 2010). Interestingly, in Japanese quails, administration of folic acid significantly increased paraoxonase and AREase activities (Gursu et al., 2004).

Moreover, our data show that the folic acid-induced increases in PON1 CMPAase and AREase activities from baseline to 3 months later are significantly and positively associated with the improvement in eGFR. Those results indicate that treatment with folic acid may improve eGFR and that these effects are mediated by increased PON1 activity, which increases lipid-associated antioxidant defenses. However, a double-blind placebo controlled randomized trial performed in 40 healthy volunteers indicated that folic acid (300 $\mu \mathrm{g} /$ daily for 12 weeks) elevated serum PON1 CMPAase enzyme activity (Voutilainen et al., 2003). In contrast, Kim et al. (2012) showed that dietary folic acid was negatively associated of AREase activity.

The possible antioxidant activity of folic acid is ascribed to its free radical scavenging properties (Joshi et al., 2001). All the radicals (thiyl radicals [RS $\left.{ }^{\circ}\right]$, peroxyl radical $\left[\mathrm{CCl}_{3} \mathrm{O}_{2}{ }^{\circ}\right]$, azide radical $\left[\mathrm{N}_{3}{ }^{\circ}\right]$, sulfate radical anions $\left[\mathrm{SO}_{4}{ }^{\circ}\right]$, bromine radical $\left[\mathrm{Br}_{2}{ }^{\circ}\right]$ and hydroxyl $\left[{ }^{\circ} \mathrm{OH}\right]$ radicals) react with folic acid under ambient conditions at an almost diffusion-controlled rate. In addition, folic acid can not only scavenge $\mathrm{RS}^{\bullet}$ but can also repair those thiols at physiological $\mathrm{pH}$. Folic acid can be converted into a coenzyme N5, N10-dimethylene5,6,7,8-tetrahydrofolate in vivo through a series of enzymatic transformations. It has purine and pyrazine type rings and two amino acids on the pyrazine ring. Its lactim form has a hydroxyl group on the purine type ring. When folic acid reacts with oxidizing free radicals, this hydroxyl group can play an important role in inhibiting the oxidation effect (Qin et al., 2014). 
The fourth major finding of this study is that Hcy levels are significantly increased from G3a to G3b to G4 and, thus, that patients belonging to stage G4 showed lower PON1 activity and higher Hcy concentrations. Our data extend those of Kerkeni et al. (2009) who reported that patients with hyperhomocysteinemia show decreased PON1 concentrations, and with the results of Choi et al. (2014) who reported that there is a negative correlation between Hcy levels and renal function. Some other studies reported that CKD is accompanied by hyperhomocysteinemia and that there are significant inverse associations between PON1 activity and Hcy (Perła-Kaja'n; Jakubowski 2012; Koyama et al., 1997). Moreover, patients with CKD show increased Hcy levels as compared with the general population (Perna et al., 2010). Van Guldener and Stehouwer (2003) demonstrated that hyperhomocysteinemia in these patients may be induced by a dysregulated Hcy metabolism in the kidneys rather than by reduced eGFR. As such, it is difficult to conclude whether Hcy or eGFR is the explanatory variable. To complicate matters, Hcy may play a mechanistic role in suppressing PON1 activity (Kennedy et al., 2013) although in our study there were no significant effects of Hcy on PON1 enzymatic activities.

The fifth major finding of this study is that folic acid significantly reduced plasma Hcy levels and the Hcy/PON1 activity ratio and that the effects of folic acid increasing PON1 activities were not mediated by changes in Hcy. Our findings extend those of previous papers reporting that treatment with folic acid $(2.5-15 \mathrm{mg} / \mathrm{d})$ may reduce Hcy concentrations in CKD (House et al., 2010; Wu, 2012; Righetti et al., 2006; Vianna et al., 2007; Cianciolo et al., 2008). Some reviews (Clase; Ki; Holden 2013; Cianciolo et al. 2017; Capelli et al. 2019) discuss that the beneficial effects of folic acid therapy may be due to its direct effects or to a reduction of hyperhomocysteinemia in patients with CKD.

Hcy may cause OS through different mechanisms including auto-oxidation, suppression of glutathione peroxidase and activation of superoxide dismutase (Cristiana; 
Nina; Elena, 2012). Moreover, Hcy contains a reactive sulfhydryl group (-SH) and, like most thiols (RSH), can undergo oxidation to the disulfide (RSSR) at a physiological pH in the presence of $\mathrm{O}_{2}$ (Jacobsen et al., 2000; Poole, 2015). The metabolism of Hcy comprises two different pathways, namely remethylation and transsulfuration. In the former pathway, methionine is regenerated through a reaction catalyzed by the enzyme methionine synthase, requiring folate and vitamin B12 as cofactors (Van Guldener; Stam; Stehouwer 2005; Obeid et al., 2016). As such, folate is involved in Hcy remethylation to methionine (Perła-Kaja'n; Jakubowski 2012). Therefore, our results that folic acid treatment may reduce Hcy concentrations, suggests that the antioxidant properties of folate contribute directly to an improved prooxidant/antioxidant in CKD patients.

In conclusion, treatment with folic acid improves eGFR and increases CMPAase and AREase activities and additionally decreases Hcy levels. The improvement in eGFR is mediated via increased PON1 activities. Folic acid treatment of CKD patients in early/intermediate stages of CKD patients improves the prooxidant (Hcy) / antioxidant (PON1 activities) ratio. Treatment with folic acid in phase G3b and G4 may reduce renal disease progression by enhancing antioxidant defenses.

\section{References}

Aslam, S. Cardiovascular disease in dialysis patients: do some antihypertensive drugs have specific antioxidant effects or is it just blood pressure reduction? Does antioxidant treatment reduce the risk for cardiovascular disease? Nephrol. Hypertens. v. 17, p. 99-105. 2008.

Atamer, A.; Bilici, A.; Yenice, N.; Selek, S.; Ilhan, N.; Atamer, Y. The Importance of Paraoxonase 1 Activity, Nitric Oxide and Lipid Peroxidation in Hepatosteatosis. J Int Med Res. 36(4):771-6. 2008. 
Barreto, F.C.; Stinghen, A.E.M.; Oliveira, R.B.; Franco, T.B.; Morenol, A.N.; Barreto, D.V.; Pecoits-Filho, R.; Drüeke, T.B.; Massy, Z.A. The quest for a better understanding of chronic kidney disease complications: an update on uremic toxins. J. Bras. Nefrol. vol.36(2). 2014. Bayrak, A.; Bayrak, T.; Bodur, E.; Kılınç, K.; Demirpençe, E. The effect of HDL-bound and free PON1 on copper-induced LDL oxidation. Chemico-Biological Interactions. 2016.

Billecke, S.; Draganov, D.; Counsell, R.; et al. Human serum paraoxonase (PON1) isozymes Q and R hydrolyze lactones and cyclic carbonate esters. Drug Metab Dispos 2000; 28: 1335 42.

Capelli, I.; Cianciolo, G.; Gasperoni, L.; Zappulo, F.; Tondolo, F.; Cappuccilli, M.; La Manna, G. Folic Acid and Vitamin B12 Administration in CKD, Why Not? Nutrients, v.11, 383. 2019.

Choi, S.T.; KIm, J.S.; Song, J. Elevated serum homocysteine levels were not correlated with serum uric acid levels, but with decreased renal function in gouty patients. J Korean Med Sci 2014; 29: 788-792

Cianciolo, G.; DE Pascalis, A.; Di lullo, L.; Ronco, C.; Zannini, C.; LA Manna, G. Folic Acid and Homocysteine in Chronic Kidney Disease and Cardiovascular Disease Progression: Which Comes First? Cardiorenal. Med. 2017, 7, 255-266

Cianciolo, G.; LA Manna, G.; Colì, L.; Donati, G.; D’Addio, F.; Persici, E.; Comai, G.; Wratten, M.; Dormi, A.; Mantovani, V.; et al. 5-methyltetrahydrofolate administration is associated with prolonged survival and reduced inflammation in ESRD patients. Am. J. Nephrol. 2008, 28, 941-948.

Clase, C.M.; Ki, V.; Holden, R.M. Water-Soluble Vitamins in People with Low Glomerular Filtration Rate or On Dialysis: A Review. Seminars in Dialysis, v.26,(5): 546-567. 2013. 
Costa, L.G.; Giordano, G.; Furlong, C.E. Pharmacological and dietary modulators of paraoxonase 1 (PON1) activity .and expression: The hunt goes on. Biochemical Pharmacology 81 (2011) 337-344.

Cristiana, F.; Nina, Z.; Elena, A. Homocysteine in red blood cells metabolism pharmacological approaches. Blood Cell: An Overview of Studies in Hematology. Cap. 3. Ed. Intech. 2012.

Daenen, K.; Andries, A.; Mekahli, D.; Van Schepdael, A.; Jouret, F.; Bammens, B. Oxidative stress in chronic kidney disease. Pediatr Nephrol. 34(6):975-991. 2019.

Doshi, S.N.; McDowell, I.F.; Moat, S.J. et al. Folate improves endothelial function in coronary artery disease: an effect mediated by reduction of intracellular superoxide? Arterioscler Thromb Vasc Biol. 2001;21:1196-1202.

Doshi, S.N.; McDowell, I.F.; Moat, S.J.; Payne, N.; Durrant, H.J.; Lewis, M.J.; Goodfellow, J. Folic Acid Improves Endothelial Function in Coronary Artery Disease via Mechanisms Largely Independent of Homocysteine Lowering. Circulation 2002, 105, 22-26.

Dounousi, E.; Papavasiliou, E.; Makedou, A.; Ioannou, K.; Katopodis, K.P.; Tselepis, A.; Siamopoulos, K.C.; Tsakiris, D. Oxidative stress is progressively enhanced with advancing stages of CKD. Am J Kidney Dis. 2006, 48(5):752-60.

Duni, A.; Liakopoulos, V.; Rapsomanikis, K.-P.; Dounousi, E. Chronic Kidney Disease and Disproportionally Increased Cardiovascular Damage: Does Oxidative Stress Explain the Burden? Oxidative Med. Cell. Longev. 2017, 2017, 1-15.

Efrat, M.; Aviram, M. Macrophage paraoxonase 1 (PON1) binding sites. Biochemical and Biophysical Research Communications, v. 376(1): 105-110, 2008.

GBD 2015. Mortality and Causes of Death Collaborators. Global, regional, and national life expectancy, all-cause mortality, and cause-specific mortality for 249 causes of death, 1980- 
2015: a systematic analysis for the Global Burden of Disease Study 2015. Lancet 2016;388:1459-544.

Gursu, M.F.; Onderci, M.; Gulcu, F.; Sahin, K. Effects of vitamin C and folic acid supplementation on serum paraoxonase activity and metabolites induced by heat stress in vivo. Nutr. Res. v.24(2):157-164. 2004.

House, A.A., Eliasziw, M., Cattran, D.C., et al. Effect of B-vitamin therapy on progression of diabetic nephropathy: a randomized controlled trial. JAMA. 2010;303(16):1603-1609. Jacobsen, D.W. Hyperhomocysteinemia and oxidative stress time for a reality check? Arterioscler Thromb Vasc Biol; 20: 1182-4. 2000.

Joshi, R.; Adhikari S; Patro BS et al. Free radical scavenging behavior of folic acid: evidence for possible antioxidant activity. Free Radic Biol Med. 2001;30:1390-1399.

Karatas, A.; Bayrak, A.; Bayrak, T.; Canakci, E. Oxidant and antioxidant mechanisms in chronic kidney disease. Ann Med Res 2019; 26(2):270-4.

KDIGO Work Group (2013). KDIGO 2012 clinical practice guideline for the evaluation and management of chronic kidney disease. Kidney Int. Suppl. 3, 1-150. 2013.

Kennedy, D.J.; Tang, W.H.W.; Fan, Y.; Wu Y.; Mann, S.; Pepoy, M.; Hazen, S.L. Diminished Antioxidant Activity of High-Density Lipoprotein- Associated Proteins Chronic Kidney Disease. Journal of the American Heart Association. 2013. p. 1-7.

Kim, D.S.; Burt, A.A.; Ranchalis, J.E.; Richter, R.J.; Marshall, J.K.; Nakayama, K.S.; Jarvik, E.R.; Eintracht, J.F.; Rosenthal, E.A.; Furlong, C.E.; Jarvik, G.P. Dietary cholesterol increases paraoxonase 1enzyme activity. Journal of Lipid Research, v. 53, 2012.

Koyama K., Yoshida A., Takeda A., Morozumi K., Fujinami T., Tanaka N. Abnormal cyanide metabolism in uraemic patients. Nephrol. Dial. Transpl. 1997;12:1622-1628.

Levey, A.S.; Bosch, J.P.; Lewis, J.B.; Greene, T.; Rogers, N.; Roth, D. A more accurate method to estimate glomerular filtration rate from serum creatinine: a new prediction 
equation. Modification of Diet in Renal Disease Study Group. Ann Intern Med. 16;130(6):461-70. 1999.

Levi, A.; Cohen, E.; Levi, M.; Goldberg, E.; Garty, M.; Krause, I. Elevated serum homocysteine is a predictor of accelerated decline in renal function and chronic kidney disease: A historical prospective study. Eur J Intern Med, v. 25, p. 951-955. 2014.

Liakopoulos, V.; Roumeliotis, S.; Bozikas, A.; Eleftheriadis T.; Dounousi, E. Antioxidant Supplementation in Renal Replacement Therapy Patients: Is There Evidence? Oxid Med Cell Longev. v. 2019. 2019.

Locatelli, F.; Canaud, B.; Eckardt, K.; Stenvinkel, P.; Wanner, C.; Zoccali, C.; Oxidative stress in end-stage renal disease: an emerging threat to patient outcome. Nephrol Dial Transplant (2003) 18: 1272-1280

Long, Y.; Nie, J. Homocysteine in Renal Injury. Kidney Dis. 2016, 2, 80-87.

Marsillach, J.; Aragonès, G.; Beltrán, R.; Caballeria, J.; Pedro-Botet, J.; Morcillo-Suárez, C.; Navarro, A.; Joven, J.; Camps, J. The measurement of the lactonase activity of paraoxonase- 1 in the clinical evaluation of patients with chronic liver impairment. Clin Biochem. 42(1-2):91-8. 2009.

Miljkovic, M.; Stefanovic, A.; Vekic, J.; Zeljkovic, A.; Gojkovic, T.; Simic-Ogrizovic, S.; Bogavac-Stanojevic, N.; Cerne, D.; Ilic, J.; Stefanovic, I.; Jelic-Ivanovic, Z.; SpasojevicKalimanovska, V.; Kotur-Stevuljevic, J. Activity of paraoxonase 1 (PON1) on HDL2 and HDL3 subclasses in renal disease. Clin Biochem. 60:52-58. 2018.

Moreira, E.G.; Boll, C.M.; Correia, D.G.; Soares, J.F.; Rigobello, C.; Maes, M. Why Should Psychiatrists and Neuroscientists Worry about Paraoxonase 1? Curr Neuropharmacol, v.17, 2019.

Nakano, E.; Higgins JA.; Powers HJ. Folate protects against oxidative modification of human LDL. Br J Nutr. 2001; 86:637-639. 
Nanayakkara, PWB.; Van Guldener, C.; Ter Wee, PM.; Scheffer, PG.; Van Ittersum, FJ.; Twisk, JW.; Teerlink, T.; Van Dorp, W.; Stehouwer, CDA. Effect of a Treatment Strategy Consisting of Pravastatin, Vitamin E, and Homocysteine Lowering on Carotid Intima-Media Thickness, Endothelial Function, and Renal Function in Patients With Mild to Moderate Chronic Kidney Disease. Arch Intern Med. 2007; 167:1262-1270.

Neuen, N.B.; Chadban, S.J.; Demaio, A.R.; Johnson, D.W.; Perkovic, V.; Chronic kidney disease and the global NCDs agenda. BMJ Glob Health. 2017.

Obeid, R.; Kirsch, SH.; Dilmann, S.; Klein, C.; Eckert, R.; Geisel, J.; Herrmann, W. Folic acid causes higher prevalence of detectable unmetabolized folic acid in serum than B-complex: a randomized trial. Eur J Nutr. 2016, 55:1021-1028.

Oberg, B.P.; McMenamin, E.; Lucas, F.L.; McMonagle, E.; Morrow, J.; Ikizler, T.A.; et al. Increased prevalence of oxidant stress and inflammation in patients with moderate to severe chronic kidney disease. Kidney Int. 2004;65:1009-16.

Perła-Kaja'n, J.; Jakubowski, H. Paraoxonase 1 protects against protein Nhomocysteinylation in humans. The FASEB Journal. v.24(3):931-936. 2010.

Perła-Kaja'n, J.; Jakubowski, H. Paraoxonase 1 and homocysteine metabolism. Amino Acids 2012; 43:1405-1417.

Perna, A.F.; Sepe, I.; Lanza, D.; Capasso, R.; Di Marino, V.; De Santo, N.G.; Ingrosso, D. The gasotransmitter hydrogen sulfide in hemodialysis patients. J. Nephrol. 2010, 23, S92S96.

Pietrzik, K.; Bailey, L.; Shane, B. Folic Acid and L-5-Methyltetrahydrofolate: Comparison of Clinical Pharmacokinetics and Pharmacodynamics. Clinical Pharmacokinetics, 49, 535548. 2010.

Poole, L.B. The Basics of Thiols and Cysteines in Redox Biology and Chemistry. Free Radic Biol Med. 0: 148-157. 2015. 
Qin, L.; Wang, W.; You, S.; Dong, J.; Zhou, Y.; Wang, J. In vitro antioxidant activity and in vivo antifatigue effect of layered double hydroxide nanoparticles as delivery vehicles for folic acid. Int J Nanomedicine. v. 9: 5701-5710. 2014

Richter, R.J.; Jarvik, G.P.; Furlong, C.E. 2008. Determination of paraoxonase 1 status without the use of toxic organophosphate substrates. Circ Cardiovasc Genet, 1 (2) 147-152. Righetti, M.; Ferrario, G.M.; Milani, S.; Serbelloni, P.; La Rosa, L.; Uccellini, M.; Sessa, A. Effects of folic acid treatment on homocysteine levels and vascular disease in hemodialysis patients. Med. Sci. Monit. 2003, 9, 19-24.

Rosenblat, M.; Volkova, N.; Ward, J.; Aviram, M. Paraoxonase 1 (PON1) inhibits monocyte-to-macrophage differentiation. Atherosclerosis, v. 219(1): 49-56, 2011.

Samouilidou, E.; Kostopoulos, V.; Liaouri, A.; Kioussi, E.; Vassiliou, K.; Bountou, E.; Grapsa, E. Association of lipid profile with serum PON1 concentration in patients with chronic kidney disease. Ren Fail. 38(10):1601-1606. 2016.

Stefanovic, A.; Ristovski-Kornic, D.; Kotur-Stevuljevic, J.; Spasojevic-Kalimanovska, V.; Vekic, J.; Miljkovic, M.; Paripovic, D. Alterations of HDL particles in children with endstage. J Med Biochem 36: 358-365, 2017.

Stępniewska, J. et al. Oxidative Stress and Antioxidative Enzyme Activities in Chronic Kidney Disease and Different Types of Renal Replacement Therapy. Current Protein and Peptide Science. v. 16, 2015.

Taylor, D.; Bhandari, S.; Seymour, A.-M.L. Mitochondrial dysfunction in uremic cardiomyopathy. Am. J. Physiol. -Ren. Physiol. 2015, 308, 579-587.

Van Guldener, C.; Stam, F.; Stehouwer, C.D. Hyperhomocysteinaemia in chronic kidney disease: Focus on transmethylation. Clin. Chem. Lab. Med. 2005, 43, 1026-1031.

Van Guldener, C.; Stehouwer, C.D. Homocysteine metabolism in renal disease. Clin. Chem. Lab. Med. 2003, 41, 1412-1417. 
Vanzin, C.S.; Mescka, C.P.; Donida, B.; Hammerschimidt, T.G.; Ribas, G.S.; Kolling, J.; Scherer, M.B.; Vilarinho, L.; Nogueira, C.; Coitinho, A.S.; Wajner, M.; Wyse, A.T.S.; Vargas, C.R. Lipid, Oxidative and Inflammatory Profile and Alterations in the Enzymes Paraoxonase and Butyrylcholinesterase in Plasma of Patients with Homocystinuria Due CBS Deficiency: The Vitamin B12 and Folic Acid Importance. Cell Mol Neurobiol, v.35:899911. 2015.

Vianna, A.C.; Mocelin, A.J.; Matsuo, T.; Morais-Filho, D.; Largura, A.; Delfino, V.A.; Soares, A.E.; Matni, A.M. Uremic hyperhomocysteinemia: A randomized trial of folate treatment for the prevention of cardiovascular events. Hemodial. Int. 2007, 11, 210-216.

Voutilainen, S.; Rissanen, T.H.; Virtanen, J.K.; Porkkala-Sarataho, E.; Kaikkonen J.; Seppänen, K.; Tuomainen, T.P.; Lehtimäki, T.; Rontu, R.; Hämelahti, P.; Penttilä, I.; Mursu J, Salonen, J.T. Folic acid supplementation increases serum paraoxonase activity: evidence from a randomized double blind oral supplementation trial in men. Current topics in nutraceutical research. v.1 (3): 175-182. 2003.

Xu, X.; Qin, X.; Li, Y.; Sun, D.; Wang, J.; Liang, M.; Wang, B.; Huo, Y.; Hou, F.F. Efficacy of Folic Acid Therapy on the Progression of Chronic Kidney Disease: The Renal Substudy of the China Stroke Primary Prevention Trial. JAMA Internal Medicine, v.176(10). 2016. Wyatt, C.M. and Spence J.D. Folic acid supplementation and chronic kidney disease progression. Kidney Int. v.90(6): 1144-1145. 2016.

Wu, C.C.; Zheng, C.M.; Lin, Y.F.; Lo, L.; Liao, M.T.; Lu, K.C. Role of homocysteine in end-stage renal disease. Clin. Biochem. 2012, 45, 1286-1294.

Yilmaz, N.; Cicek, HK.; Celik, A.; Davutoglu, V. Diagnostic value of bilirubin concentrations compared with novel and traditional biomarkers in atherosclerosis with coronary artery disease. Saudi Med J 2006; 27: 1262-4. 
Yilmaz, N. Relationship between paraoxonase and homocysteine: crossroads of oxidative diseases. Arch Med Sci, 2012. 138-153.

Zha, Y.; Qian, Q. Protein Nutrition and Malnutrition in CKD and ESRD. Nutrients 2017, 27, 208. 
Table 1: Comparison of socio-demographic, clinical and baseline biomarker data between chronic kidney disease patients treated with or without folic acid.

\begin{tabular}{|c|c|c|c|c|c|}
\hline \multicolumn{6}{|c|}{$\begin{array}{l}\text { Table 1: Comparison of socio-demographic, clinic } \\
\text { patients treated with or without folic acid. }\end{array}$} \\
\hline Variables & $\begin{array}{l}\text { No Folic acid } \\
\text { Treatment } \\
(n=47)\end{array}$ & $\begin{array}{l}\text { Folic acid } \\
\text { Tratment } \\
(\mathrm{n}=66)\end{array}$ & $\mathbf{F} / \mathbf{X}^{2}$ & df & $\mathbf{P}$ \\
\hline Age (years) & $67.2(12.4)$ & $66.2(13.1)$ & 0.17 & $1 / 111$ & 0.678 \\
\hline $\operatorname{Sex}(\mathrm{F} / \mathrm{M})$ & $19 / 28$ & $30 / 36$ & 0.28 & 1 & 0.595 \\
\hline Ethnicity $(\mathrm{C} / \mathrm{nC})$ & $39 / 8$ & $59 / 7$ & 0.98 & 1 & 0.322 \\
\hline BMI $\left(\mathrm{kg} / \mathrm{m}^{2}\right)$ & $29.3(5.4)$ & $29.1(5.1)$ & 0.03 & $1 / 110$ & 0.870 \\
\hline Diabetes (Y/N) & $23 / 24$ & $29 / 37$ & 0.77 & 1 & 0.599 \\
\hline Statin use (Y/N) & $10 / 37$ & $9 / 57$ & 1.15 & 1 & 0.284 \\
\hline HAA (Y/N) & $16 / 29$ & $18 / 47$ & 0.77 & 1 & 0.380 \\
\hline AS A use (Y/N) & $29 / 16$ & $35 / 30$ & 1.23 & 1 & 0.268 \\
\hline Smoking (Y/N) & $4 / 43$ & $4 / 62$ & 0.25 & 1 & 0.617 \\
\hline $\mathrm{SP}(\mathrm{mm} \mathrm{Hg})$ & $120.6(16.1)$ & $129.0(18.6)$ & 7.36 & $1 / 111$ & 0.008 \\
\hline $\mathrm{DP}(\mathrm{mm} \mathrm{Hg})$ & $82.8(13.5)$ & $83.2(12.7)$ & 0.03 & $1 / 111$ & 0.867 \\
\hline Folic acid $(\mathrm{mg} / \mathrm{mL})$ & $7.87(3.0)$ & $8.71(3.66)$ & 1.81 & $1 / 110$ & 0.182 \\
\hline eGFR (mL/min/, 1.73m²) & $36.1(12.7)$ & $33.8(12.5)$ & 0.98 & $1 / 111$ & 0.325 \\
\hline CMPAase (U/mL) & $28.6(10.3)$ & $28.1(10.7)$ & 0.07 & $1 / 111$ & 0.787 \\
\hline AREase $(\mathrm{U} / \mathrm{mL})$ & $182.5(44.5)$ & $178.9(46.6)$ & 0.17 & - & 0.679 \\
\hline PON genotype (QQ/QR/RR) & $22 / 23 / 1$ & $29 / 31 / 6$ & $\Psi=0.14$ & - & 0.330 \\
\hline GRF groups & $16 / 16 / 15$ & $29 / 23 / 14$ & 1.91 & 2 & 0.386 \\
\hline Homocysteine $(\mathrm{mmol} / \mathrm{L})$ & $18.79(12.28)$ & $18.88(5.50)$ & 0.98 & $1 / 110$ & 0.325 \\
\hline Vitamin B12 (mg/mL) & $408.6(209.0)$ & $369.5(235.3)$ & 0.81 & $1 / 107$ & 0.369 \\
\hline
\end{tabular}




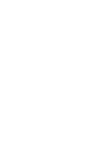

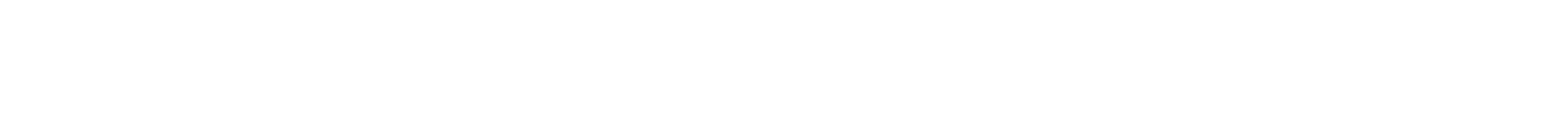

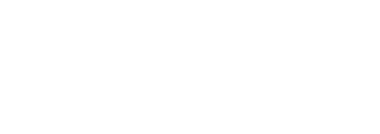

\begin{abstract}
Preprints (www.preprints.org) | NOT PEER-REVIEWED | Posted: 31 March $2020 \quad$ doi:10.20944/preprints202003.0472.v1
All results are shown as mean (SD). BMI: body mass index; ASA: acetylsalicylic acid; HAA: arterial hypertension; SP: systolic
blood pressure, DP, diastolic blood pressure, GRF: glomerular react filtration; CMPAase: 4-(chloromethyl)phenyl acetate
activity; AREase: arylesterase activity; GFR: glomerular filtration rate. *Processed in Ln transformation.
Preprints (www.preprints.org) | NOT PEER-REVIEWED | Posted: 31 March $2020 \quad$ doi:10.20944/preprints202003.0472.v1
All results are shown as mean (SD). BMI: body mass index; ASA: acetylsalicylic acid; HAA: arterial hypertension; SP: systolic
blood pressure, DP, diastolic blood pressure, GRF: glomerular react filtration; CMPAase: 4-(chloromethyl)phenyl acetate
activity; AREase: arylesterase activity; GFR: glomerular filtration rate. *Processed in Ln transformation.

Preprints (www.preprints.org) | NOT PEER-REVIEWED | Posted: 31 March $2020 \quad$ doi:10.20944/preprints202003.0472.v1
All results are shown as mean (SD). BMI: body mass index; ASA: acetylsalicylic acid; HAA: arterial hypertension; SP: systolic
blood pressure, DP, diastolic blood pressure, GRF: glomerular react filtration; CMPAase: 4-(chloromethyl)phenyl acetate
activity; AREase: arylesterase activity; GFR: glomerular filtration rate. *Processed in Ln transformation.

Preprints (www.preprints.org) | NOT PEER-REVIEWED | Posted: 31 March $2020 \quad$ doi:10.20944/preprints202003.0472.v1
All results are shown as mean (SD). BMI: body mass index; ASA: acetylsalicylic acid; HAA: arterial hypertension; SP: systolic
blood pressure, DP, diastolic blood pressure, GRF: glomerular react filtration; CMPAase: 4-(chloromethyl)phenyl acetate
activitv.
\end{abstract}


Table 2: Effect of folic acid treatment on glomerular filtration rate (GFR), paraoxonase (PON)1 CMPAase and AREase activities, and homocysteine: results of GEE analyses, repeated measures.

\begin{tabular}{|c|c|c|c|c|c|c|c|c|c|}
\hline \multirow{2}{*}{ Variables } & \multirow{2}{*}{\begin{tabular}{|l|} 
Active \\
Treatment
\end{tabular}} & \multirow{2}{*}{ Basal } & \multirow{2}{*}{ Post treatment } & \multicolumn{2}{|c|}{ Treatment } & \multicolumn{2}{|l|}{ Time } & \multicolumn{2}{|c|}{ Treatment $x$ time } \\
\hline & & & & $\mathbf{F}$ & $\mathbf{p}$ & $\mathbf{F}$ & $\mathbf{P}$ & $\mathbf{F}$ & $\mathbf{p}$ \\
\hline $\begin{array}{l}\text { eGFR }(\mathrm{mL} / \mathrm{min} / \text {, } \\
\left.1.73 \mathrm{~m}^{2}\right)\end{array}$ & $\begin{array}{l}\text { No } \\
\text { Yes }\end{array}$ & $\begin{array}{l}36.5(0.7) \\
36.8(0.5)\end{array}$ & $\begin{array}{l}37.4(1.1) \\
42.3(1.6)^{*}\end{array}$ & 6.21 & 0.013 & 8.70 & 0.003 & 4.52 & 0.034 \\
\hline Folic Acid $(\mathrm{mg} / \mathrm{mL})$ & \begin{tabular}{|l|} 
No \\
Yes \\
\end{tabular} & \begin{tabular}{|l|}
$7.79(0.49)$ \\
$8.55(0.56)$ \\
\end{tabular} & $\begin{array}{l}9.04(0.81) \\
37.77(3.07)^{* * *} \\
\end{array}$ & 100.1 & $<0.001$ & 126.6 & $<0.001$ & 88.83 & $<0.001$ \\
\hline $\begin{array}{l}\text { PON1 CMPAase } \\
(\mathrm{U} / \mathrm{mL})\end{array}$ & $\begin{array}{l}\text { No } \\
\text { Yes }\end{array}$ & $\begin{array}{l}32.7(1.4) \\
34.4(1.6)\end{array}$ & $\begin{array}{l}34.4(1.6) \\
39.1(1.5)^{*}\end{array}$ & 1.38 & 0.240 & 9.14 & 0.003 & 3.84 & 0.05 \\
\hline PON1 AREase $(\mathrm{U} / \mathrm{mL})$ & $\begin{array}{l}\text { No } \\
\text { Yes }\end{array}$ & $\begin{array}{l}193.0(6.4) \\
189.5(6.1)\end{array}$ & $\begin{array}{l}189.9(7.9) \\
221.9(5.6)^{* *}\end{array}$ & 3.79 & 0.052 & 9.16 & 0.002 & 13.46 & $<0.001$ \\
\hline $\begin{array}{l}\text { CMPAase + AREase } \\
\text { (z scores) }\end{array}$ & $\begin{array}{l}\text { No } \\
\text { Yes }\end{array}$ & $\begin{array}{l}0.25(0.18) \\
0.08(0.17)\end{array}$ & $\begin{array}{l}0.32(0.21) \\
1.38(0.17)^{* * *}\end{array}$ & 4.96 & 0.026 & 17.70 & $<0.001$ & 14.26 & $<0.001$ \\
\hline $\begin{array}{l}\text { Homocysteine } \\
(\mathrm{mmol} / \mathrm{L})\end{array}$ & $\begin{array}{l}\text { No } \\
\text { Yes }\end{array}$ & $\begin{array}{l}18.79(1.78) \\
18.87(0.68)\end{array}$ & $\begin{array}{l}16.87(0.74) \\
14.49(0.46)^{* *}\end{array}$ & 0.64 & 0.423 & 18.02 & $<0.001$ & 8.23 & 0.004 \\
\hline $\begin{array}{l}\text { PROOX / ANTIOX } \\
\text { ratio }\end{array}$ & $\begin{array}{l}\text { No } \\
\text { Yes }\end{array}$ & $\begin{array}{l}0.069(0.213) \\
0.251(0.163)\end{array}$ & $\begin{array}{l}-0.117(0.181) \\
-1.349(0.166)^{* * *}\end{array}$ & 3.32 & 0.069 & 34.20 & $<0.001$ & 20.83 & $<0.001$ \\
\hline Vitamin B12 (mg/mL) & $\begin{array}{l}\text { No } \\
\text { Yes }\end{array}$ & $\begin{array}{l}408.6(30.2) \\
372.6(29.8)\end{array}$ & $\begin{array}{l}421.3(50.0) \\
504.9(82.9)\end{array}$ & 0.09 & 0.765 & 0.34 & 0.558 & 2.60 & 0.107 \\
\hline
\end{tabular}


Results are shown as estimated marginal means (SE) obtained by GEE analyses after covarying for PON1 genotype and GFR groups. CMPAase + AREase: index
of overall PON1 enzyme activity computed as z value of CMPAase (zCMPAase) + z AREase. PROOX/ANTIOX: computed as z homocysteine - z(zCMPAase +
zAREase). ${ }^{*} \mathrm{p}<0.05,{ }^{*} \mathrm{p}<0.01, * * * \mathrm{p}<0.001$ : significantly different from the other three conditions.

Results are shown as estimated marginal means (SE) obtained by GEE analyses after covarying for PON1 genotype and GFR groups. CMPAase + AREase: index
of overall PON1 enzyme activity computed as z value of CMPAase (zCMPAase) + z AREase. PROOX/ANTIOX: computed as z homocysteine - $\mathrm{z}(\mathrm{zCMPAase}+$
zAREase). $* \mathrm{p}<0.05, * * \mathrm{p}<0.01, * * * \mathrm{p}<0.001$ : significantly different from the other three conditions.

Results are shown as estimated marginal means (SE) obtained by GEE analyses after covarying for
of overall PON1 enzyme activity computed as z value of CMPAase (zCMPAase) $+\mathrm{z}$ AREase. PRO
zAREase). ${ }^{*} \mathrm{p}<0.05, * * \mathrm{p}<0.01, * * * \mathrm{p}<0.001$ : significantly different from the other three conditions. 
Table 4. Differences in PON1 activity and homocysteine levels between study groups based on glomerular filtration

rate cut-off values.

\begin{tabular}{|c|c|c|c|c|c|c|}
\hline \multirow{2}{*}{ Variables } & \multicolumn{3}{|l|}{ GFR groups } & \multirow{2}{*}{$\mathbf{F}$} & \multirow{2}{*}{ df } & \multirow{2}{*}{$\mathbf{p}$} \\
\hline & G4 ${ }^{\mathrm{A}}$ & $\mathbf{G 3 b}^{\mathrm{B}}$ & G3a ${ }^{C}$ & & & \\
\hline PON1 CMPAase (U/mL) & $31.3(0.9)^{\mathrm{B}, \mathrm{C}}$ & $35.8(1.3)^{\mathrm{A}}$ & $36.5(1.5)^{\mathrm{A}}$ & 15.7 & 2 & 0.001 \\
\hline PON1 AREase (U/mL) & $183.4(5.7)^{\mathrm{B}, \mathrm{C}}$ & $205.0(7.1)^{\mathrm{A}}$ & $207.9(7.0)^{\mathrm{A}}$ & 10.25 & 2 & 0.006 \\
\hline CMPAase + AREase (z scores) & $-0.058(0.091)^{\mathrm{B}, \mathrm{C}}$ & $0.468(0.119)^{\mathrm{A}}$ & $0.559(0.115)^{\mathrm{A}}$ & 22.32 & 2 & $<0.001$ \\
\hline Homocysteine (mmol/L) & $20.58(1.13)^{\mathrm{B}, \mathrm{C}}$ & $16.86(0.58)^{\mathrm{A}, \mathrm{C}}$ & $15.54(0.65)^{\mathrm{A}, \mathrm{B}}$ & 35.36 & 2 & $<0.001$ \\
\hline PROOX/ANTIOX ratio & $0.577(0.164)^{\mathrm{B}, \mathrm{C}}$ & $-0.452(0.169)^{\mathrm{A}, \mathrm{C}}$ & $-0.998(0.184)^{\mathrm{A}, \mathrm{B}}$ & 46.90 & 2 & $<0.001$ \\
\hline
\end{tabular}

All results of GEE analyses with the estimated marginal means (SE) after adjustment for treatment, time, PON1 genotype, age, sex and body mass index. CMPAase + AREase: index of overall PON1 enzyme activity computed as z value of CMPAase (zCMPAase) $+\mathrm{z}$ AREase. PROOX/ANTIOX: computed as z homocysteine - z(zCMPAase + zAREase).

PROOANTIOX: computed as $z$ homocysteine $-z(z \mathrm{CMPA}$ ase $+z$ AREase). 
Table 5. Three-way interaction with effects of treatment, time and GFR groups on the integrated index of CMPAase + AREase activities.

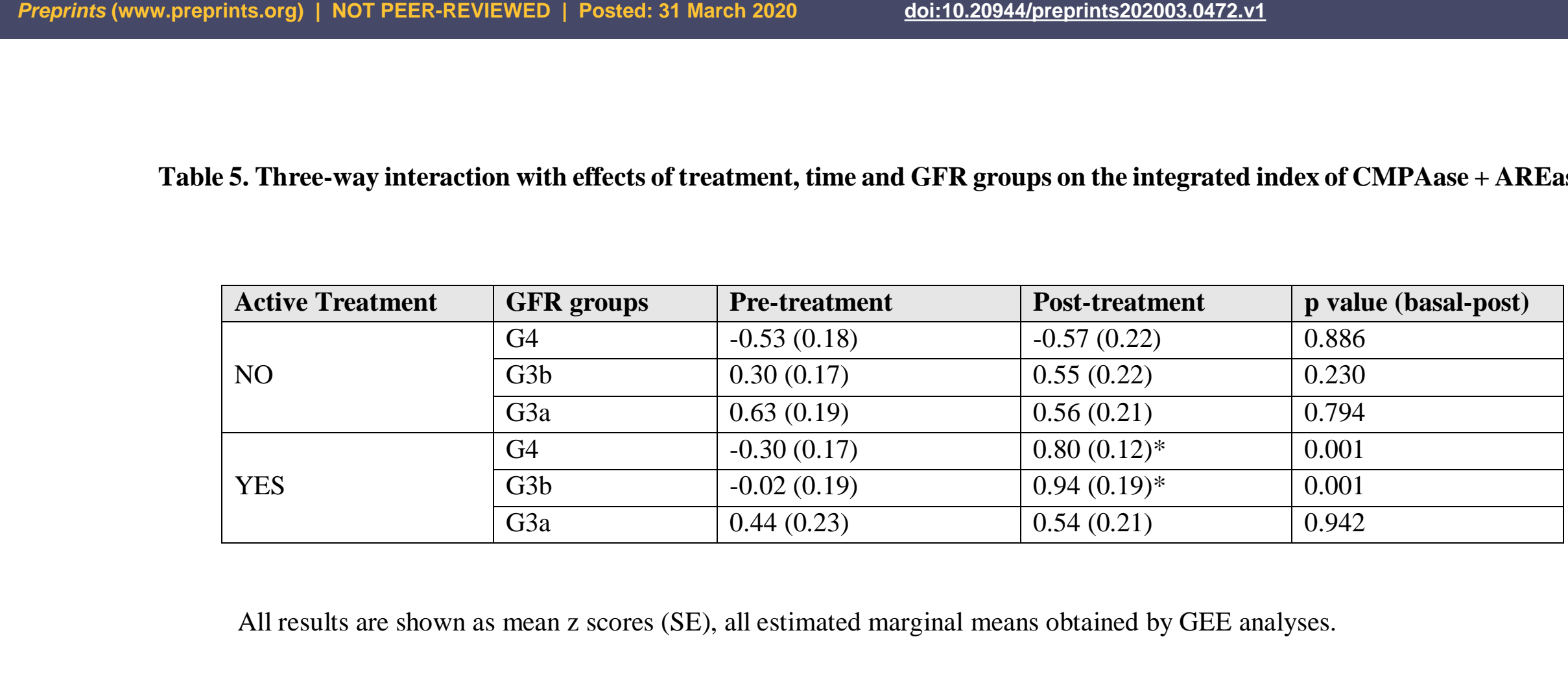

All results are shown as mean z scores (SE), all estimated marginal means obtained by GEE analyses. 